\title{
Book report \\ Pocket Guide to Perioperative and Critical Care Echocardiography
}

\author{
Jan Poelaert \\ University Hospital Gent, Cardiac Anaesthesia \& Postoperative Cardiac Surgical ICU, 5 K12 IE, \\ De Pintelaan 185, B-9000 Gent, Belgium
}

Corresponding author: Jan Poelaert, jan.poelaert@ugent.be

Published: 9 August 2007

Critical Care 2007, 11:312 (doi:10.1186/cc5979)

This article is online at http://ccforum.com/content/11/4/312

(C) 2007 BioMed Central Ltd

Royse C, Donnan G, Royse A (Eds): Pocket Guide to Perioperative and Critical Care Echocardiography. McGraw-Hill; 2006. 250 pp. ISBN $0074716115 / 9780074716113$

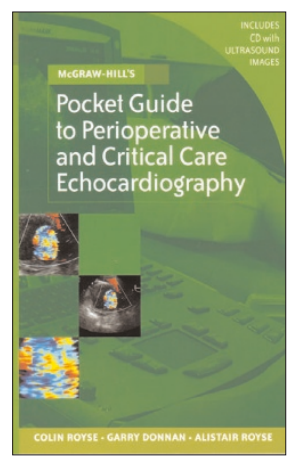

This pocket guide on perioperative echocardiography discusses various issues on echocardiography and Doppler imaging, a diagnostic and monitoring technique that is used increasingly in the perioperative and intensive care unit setting.

The setup of the pocket guide is clear and straightforward. The sequence of the different topics is built up logically and step by step. Each chapter begins with learning objectives, informing the reader immediately what is to be found in that chapter.

The first chapter deals with general views on how to use and implement echocardiography in a perioperative setting. The following two sections encompass the technology of the echocardiograph and probes. Quality of imaging and organization of echo-imaging is the topic of another chapter.

The authors provide careful instruction to the reader on what different anatomical characteristics are, how they can be obtained and how these features should be viewed in terms of a three-dimensional construction from two-dimensional images. Transoesophageal imaging and transthoracic echocardiographic imaging are discussed in separate chapters, which also provide the official terminology.

The chapter on Doppler imaging provides an overview of such basic issues as the Doppler effect and rapidly progresses to information on how to assess pressures with Doppler. This section forms the basis for the following chapter, on the assessment of haemodynamics. Various self-explanatory schemes guide the reader through decision making in this difficult issue. In addition, tables are well organized to illustrate decision making on hypovolaemia, assessment of fluid responsiveness and evaluation of ventricular function.

Ventricular function is also covered later, encompassing both systolic and diastolic function of the left ventricle. Again, various diagrams provide illustrative information.
Subsequent chapters discuss the most frequent problems of valvular pathology and provide information on problems with great vessels, and a further chapter discusses pericardial pathology from an echocardiographic point of view. In particular, the important issue of pericardial tamponade is elaborated extensively in clear diagrams.

Regional anaesthesia and vascular access - two hot topics at the moment - are included in the book's later sections, and the information presented is important for general anaesthetists. Both issues are exemplified in several schemes. The final chapter discusses which items should be included when training and accreditation is organized.

The booklet includes a small CD-Rom including several clips of most important issues, sorted by chapter.

The pocket guide does not have the aim of including all facets of echocardiography. For example, tissue Doppler imaging is discussed only very briefly. In addition, other issues such as valvular pathology are summarized, although the most important problems are discussed. Some iconography could have been more specific and illustrative, as modern echographs often offer pretty visualizations. Real images could have replaced the presented schematic representations in the chapter on regional anaesthesia and vascular access. In summary, this booklet is a good pocket guide for anaesthetists or critical care physicians who deal with echocardiography and an echographic approach to haemodynamic follow-up and problem solving.

\section{Competing interests}

The author declares that they have no competing interests. 